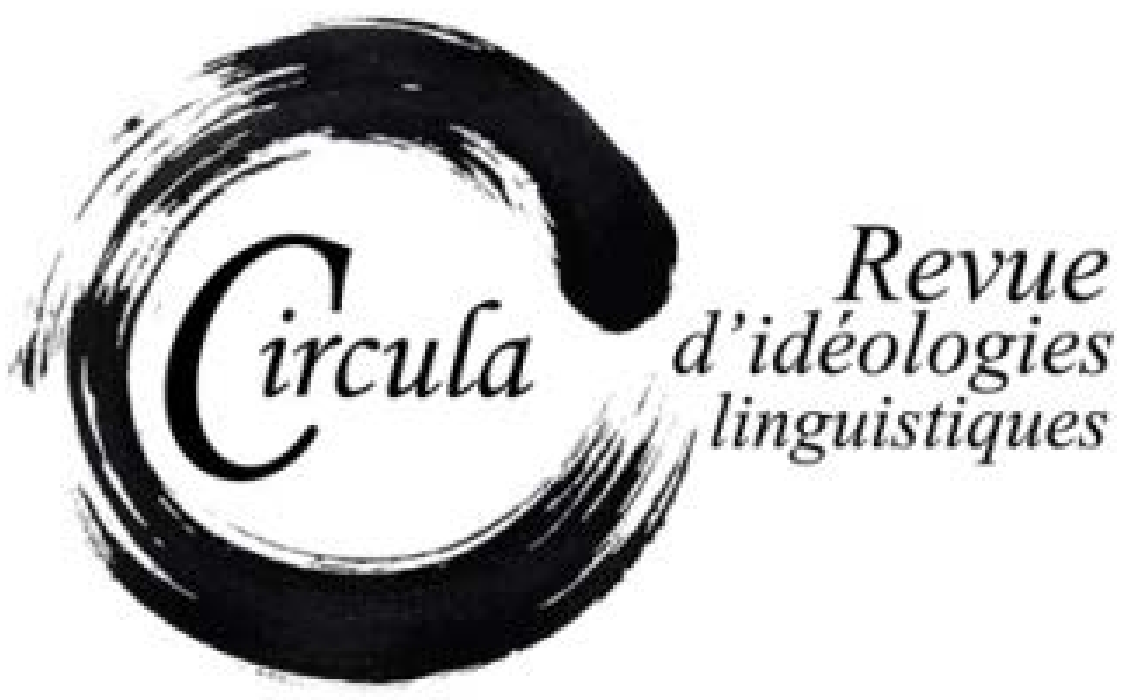

TITRE: IDEOLOGIA LINGUISTICA DELLA STAMPA NELLA CAMPAGNA ELETTORALE PER LE POLITICHE DEL 2013 Auteur(s): FABIO RuggIano, UniVersitÀ di Messina

RevUe: CirCula, NUMÉRO 1, PAGES 216-234

ISSN: 2369-6761

DiRecteurs: Wim Remysen et SABINE SCHWARZE

URI: HTTP://HDL.HANDLE.NET/11143/8001

DOI: $10.17118 / 11143 / 8001$ 


\section{Ideologia linguistica della stampa nella campagna elettorale per le politiche del 2013}

Fabio Ruggiano, Università di Messina fruggiano@unime.it

Riassunto: Questo contributo si propone di indagare il ruolo che la stampa attribuisce al linguaggio che usa, quindi a sé stessa, nel processo dell'informazione. L'attribuzione di tale ruolo emergerà dall'analisi di articoli apparsi su giornali on line di rilievo nazionale. Di questi articoli, alcuni riportano giudizi, espliciti e impliciti, su vari usi linguistici nella comunicazione politica per la campagna delle elezioni politiche del 2013. Altri riusano lessico specialistico della politica. La tesi di fondo del contributo è che gli scopi comunicativi della stampa italiana non sono diversi da quelli dei politici: tanto i politici quanto la stampa si sforzano di convincere il pubblico facendo appello in vari modi alla sua emotività, a discapito della logica e della fattualità.

Parole chiave: comunicazione politica; campagna elettorale; politica e stampa

Abstract: This paper investigates the role the press grants to the language it uses in the process of information. The nature of this role will emerge from the analysis of articles published on online newspapers of national importance. Some of these articles report judgments, explicit and implicit, on various linguistic uses in political communication during the campaign for the general election of 2013; others reuse specialized political vocabulary. The basic thesis of the paper is that the communicative purposes of the Italian press are no different from those of politicians: both politicians and the press strive to convince the public in various ways by appealing to their emotions, at the expense of logic and factuality.

Key words: political communication; electoral campaign; politics and press 


\section{Introduzione}

Il presente contributo si basa sul dibattito che si è sviluppato in Italia nel periodo dal 28 gennaio al 24 febbraio 2013, ovvero la campagna per le elezioni politiche 2013. La ricerca si spinge anche oltre, fino all'inizio del dicembre 2012, visto che, di là dall'inizio ufficiale della campagna elettorale, il gioco delle alleanze e le previsioni sulla legislazione successiva erano già iniziati settimane prima.

Il materiale è stato ricavato da diversi giornali on line di respiro nazionale ${ }^{1}$, che in Italia rappresentano attualmente uno dei più diffusi canali di informazione ${ }^{2}$. Sempre meno frequenti e frequentati sono, infatti, i contatti diretti tra l'elettorato e gli eletti, i comizi, le assemblee, le convention; mentre i social network sono finora stati usati da parte dei leader e dei partiti per rilanciare temi già discussi in televisione, con lo stesso linguaggio e con le stesse modalità. Come spiegano Antenore, Di Giammaria e Gennaro (2013):

Twitter si è ritagliato un ruolo ancillare nell'ecosistema dell'informazione politica ufficiale. Contestualmente [...], la piattaforma ha funzionato quale poderosa cassa di risonanza, spesso dissenziente, delle suggestioni provenienti dallo schermo televisivo senza riuscire, almeno sul fronte dei temi della campagna, a scalfirne l'agenda, anzi «assorbendone» in qualche modo la logica di funzionamento. (Antenore, Di Giammaria e Gennaro, 2013: 192)

Dal momento che l'indagine riguarda i principi ispiratori dell'attività giornalistica in Italia, e non le caratteristiche distintive dei media, i risultati sono stati considerati applicabili alla stampa in generale.

Sono stati selezionati soprattutto interventi di giornalisti a commento di dichiarazioni di politici, per rilevare in questi commenti le opinioni espresse sul linguaggio della propaganda. In alcuni casi, le opinioni non sono espresse in forma esplicita, ma emergono in seguito all'analisi linguistica degli articoli³. Il materiale è stato vagliato all'interno del quadro della linguistica testuale, a cui sono state associate categorie provenienti dalla discourse analysis, dalla pragmalinguistica e della sociologia della comunicazione, per evidenziare il legame tra produzione testuale e scopi comunicativi impliciti.

1. Le testate rainews24.rai.it, tg24.sky.it e rai1.rai.it, in quanto siti web, sono qui considerate alla stregua di giornali on line.

2. Al netto delle differenti abitudini legate all'età della popolazione, Human Highway/Liquida (2011) rivela che già nel 2011 Internet (giornali on line, aggregatori di notizie, blog, social network) era il mezzo più usato dagli italiani per l'approfondimento di notizie, superando tanto i giornali cartacei quanto la televisione. La televisione era ancora in testa come mezzo attraverso cui gli italiani avevano il primo contatto con le notizie. Questi dati sono in qualche modo confermati da Censis (2013), secondo cui nel 2013 i telegiornali sono ancora di gran lunga il mezzo di informazione più consultato (86,4\% della popolazione), seguiti dai giornali radio e dai motori di ricerca (46,4\%). Questi ultimi, non è da sottovalutare, restituiscono ai primi posti tra i risultati proprio pagine di giornali on line. Oltre ai motori di ricerca, secondo Censis (2013), risorse web molto usate sono i siti web di informazione (22,6\%) e i siti web dei telegiornali (12,9\%). Coloro che consultano direttamente i giornali on line, invece, sono il $20 \%$ della popolazione.

3. Le opinioni sulla lingua non devono essere per forza esplicitate, ma sono, piuttosto, «Representations, whether explicit or implicit, that construe the intersection of language and human beings in a social world» (Woolard, 1998: 3). 


\section{La comunicazione pubblica nei media e tra i media}

Stando ai giudizi espressi sulla stampa, l'appello all'emotività a discapito dei contenuti sembra essere stato lo scopo comunicativo più decisamente ricercato dai politici nella campagna elettorale 2013. Le strategie più comuni per raggiungere questo scopo sono state: la frammentarietà della proposta politica, con la riduzione del discorso ad una serie di slogan, la vaghezza nell'esposizione dei punti del programma, declinata in vari modi, l'aggressività verbale. Rispetto a questi aspetti della comunicazione politica, i giudizi dei giornali sono stati di vario genere, di condanna, neutrali, elogiativi.

Un articolo non firmato, ilsole24ore.com (20 gennaio 2013), elenca varie espressioni ingiuriose, o comunque irrispettose, che i politici si sono scambiati durante la campagna:

(1) Berlusconi pifferaio, Monti mascalzone, Bersani Gargamella. A un mese dalle elezioni è una campagna piena di insulti.

In campagna elettorale la tensione, inevitabilmente, sale. E dal confronto allo scontro verbale il passo è breve. L'insulto, insomma, è sempre dietro l'angolo. [...]

Agli attacchi verbali tra i contendenti, gli elettori ci sono ormai abituati, da almeno quindici anni. E la tornata elettorale di questo 2013 non fa eccezione. Un contributo corposo alla campagna elettorale portata avanti a colpi di battute, colpi bassi, calembour e parolacce lo fornisce proprio il leader del Movimento 5 stelle. L'elenco è lungo e i bersagli non si contano più $[\ldots]$.

Ai livelli di Grillo, il Cavaliere non ci arriva, però l'ex premier non ha fatto mancare, in queste settimane di «tour» tra tv pubbliche e private, qualche regalo verbale agli avversari, tanto per mettere un po' di pepe alla campagna elettorale. [...]

Le sue stilettate agli avversari conservano comunque un certo aplomb [...].

[...] ha detto Monti, che ha riservato al leader del Pdl un altro colpo basso, inferto con i guanti bianchi $[\ldots]$

E a sette anni di distanza è ricomparso sulla scena il «coglioni» che il Cavaliere aveva graziosamente elargito nel 2006 a quegli italiani che votavano a sinistra. [...] E al pubblico in studio che ha cominciato a fischiarlo, ha concesso il bis: «l coglioni siete voi». L'ultima perla del Cavaliere è il «matto» con cui ha etichettato il suo bersaglio principale, Monti [...].

Tra i protagonisti di questa campagna elettorale c'è il leader di Sel Nichi Vendola [...]. [...] il consigliere comunale del Pdl di Vigevano, Andrea Di Pietro, ha sparato dal suo profilo facebook un «Vendola è viscido quanto la vaselina che usa» [sic].

Elegante anche l'affondo [...] un «vaffa» omofobo, condito con l'accostamento a Tony Blair [...]. In punta di penna, o meglio di «tastiera» [sic], anche l'invettiva di Giuseppe Ripa, assessore pidiellino al traffico a Lecce, che ha colpito il leader di Sel con un uno-due: «Signorina» e «gay disturbato». Poi, però, si è dimesso. 
[...] rimanere ai margini del ring verbale sul quale se le danno di santa ragione gli altri contendenti. E non ci sarebbe niente di male, in fin dei conti: anche il leader più potente del mondo il presidente degli Stati Uniti Barack Obama, si è lasciato scappare qualche insulto nella campagna per le presidenziali (poi vinte) dello scorso anno [...].

Monti rivendica la svolta rispetto al passato e a enfatizzare politicamente il concetto ci pensa il presidente della Camera e leader Fli Gianfranco Fini [...].

A Porta a porta, infine, incrociano le lame il segretario del Pdl Angelino Alfano e il leader Udc Pier Ferdinando Casini [...].

L'articolo, dopo aver lasciato intendere che in campagna elettorale sia normale passare dalla dialettica all'insulto, inquadra l'elenco di insulti all'interno di una cornice costruita per essere in linea con il tono del tema trattato. Mi soffermo su tre tratti compositivi del testo, riconducibili allo stile brillante (Dardano, 1994: 218): l'ironia, la metaforizzazione dei concetti, la mimesi del parlato sul piano diegetico. I primi due sono strettamente legati, visto che molte metafore hanno una coloritura ironica.

Raggruppo le metafore secondo i domini del reale da cui sono tratte le immagini:

1. Lo sport, in particolar modo la boxe e la scherma, e la guerra («colpi bassi», «bersagli», «elegante affondo», «stilettate», «un colpo basso inferto con i guanti bianchi», «ha sparato dal suo profilo facebook», «in punta di penna, o meglio di tastiera», «ha colpito con un unodue», «ring verbale sul quale se le danno di santa ragione», «incrociano le lame»);

2. Lo spettacolo, significativamente ad inquadrare Berlusconi («ricomparso sulla scena», «ha concesso il bis»);

3. La cucina («mettere un po' di pepe», «"vaffa” condito»);

4. Lo spazio («il passo è breve», «è dietro l’angolo»);

5. I beni materiali, anche questi ruotanti intorno a Berlusconi («regalo verbale», «aveva graziosamente elargito», «l'ultima perla»).

Il dominio di gran lunga più sfruttato è, dunque, quello dello sport e della guerra; si tratta dello stesso bacino da cui il discorso politico trae il grosso del suo materiale immaginario, come già rilevato da Gualdo e Dell'Anna (2004):

Metafore sportive e metafore belliche sono aspetti di una stessa realtà, poiché consegnano al destinatario del messaggio la comune immagine di opposizione tra fazioni, di gara, di vittorie e di sconfitte. In modo analogo le immagini della competizione sportiva e militare sono trattate dai media, che ricercano l'elemento notiziabile proprio nella dimensione agonistica, dove è difficile dire se si tratti di sport o di guerra. (Gualdo e Dell’Anna, 2004: 58-59) 
Per quanto riguarda la mimesi del parlato, spicca per numero di occorrenze la struttura della dislocazione a sinistra: «agli attacchi verbali [...] gli elettori ci sono ormai abituati»; «un contributo corposo [...] lo fornisce proprio il leader»; «ai livelli di Grillo, il Cavaliere non ci arriva»; «a enfatizzare politicamente il concetto ci pensa il presidente». Agli esempi possiamo aggiungere anche questo, molto meno marcato: «tra i protagonisti di questa campagna elettorale c’è il leader».

Il lessico è a tratti ricercato e persino forbito (a parte le parole con cui sono costruite alcune metafore, contributo corposo, calembour, stilettate, aplomb, colpo basso, inferto, graziosamente elargito, rivendica, enfatizzare), per creare un contrasto con l'abbassamento del tono ottenuto tramite la sintassi. L'intreccio di alto a basso è anch'esso uno strumento dell'ironia.

L'apparente familiarità ottenuta tramite la mimesi del parlato, così come la forte carica ironica e metaforica, sono strategie che rivelano l'adesione di fondo, se non ai modi, sicuramente agli scopi del linguaggio oggetto dell'articolo: abbassare la complessità concettuale della comunicazione in favore dell'emotività diretta, accattivare, suscitare e mantenere l'interesse del pubblico, stimolandone la partecipazione irriflessa.

Gli artifici retorici, infatti, producono, come argomenta Loporcaro (2005: 71-72), un'impressione superficiale di familiarità e semplicità, ma nella sostanza sostituiscono il giudizio ai fatti. Aumentando l'ambiguità a discapito della precisione dei contenuti, i giornali allineano il proprio linguaggio a quello politico, che «has to consist largely of euphemism, question-begging and sheer cloudy vagueness. [...] Such phraseology is needed if one wants to name things without calling up mental pictures of them» (Orwell, 1946: 262) .

Un’analisi articolata, questa volta del linguaggio del solo Mario Monti, è fornita da Simone Regazzoni (Regazzoni: 4 gennaio 2013):

(2) Il nuovo linguaggio del professore scatenato

E' lo stesso uomo. Eppure il Professor Mario Monti, che fino a ieri usava un linguaggio tecnico, sobrio e pacato, è oggi, come il Django di Tarantino, unchained, «scatenato», capace di dichiarare dagli schermi Rai durante la trasmissione Uno Mattina: «Tagliare le ali in genere è una brutta espressione, ma se intendiamo le ali estreme credo che sia una buona cosa». Una metafora forte ma vaga? No, piuttosto un attacco mirato. Tra le ali da «tagliare» ci sono sia quelle del Pdl, sia quelle del Pd, che Monti chiama per nome: Cgil, Vendola, Fassina.

[...] Ma Monti non si ferma qui, rincara la dose, chiedendo a Bersani di «silenziare» le frange estreme. Ancora un'espressione forte: perché chiede di ridurre al silenzio, di togliere la parola. Non è un caso che il verbo «silenziare» abbia anche un significato bellico, come ricorda il Devoto Oli: «Rendere inefficiente una postazione nemica».

4. «Deve essere costituito in larga parte di eufemismi, argomentazioni che danno per dimostrato ciò che intendono dimostrare e una mera fumosa imprecisione. [...] Questa fraseologia è necessaria se si vuole nominare le cose senza evocarne un'immagine mentale.» (Trad. F. R.) 
Ai vertici del Pd, vittima di una comunicazione che oscilla tra monotonia e comicità involontaria delle metafore bersaniane, il linguaggio del New Monti, il Monti scatenato nelle vesti di leader di coalizione, deve essere suonato duro come il destro-sinistro con cui Ali, nella notte di Kinshasa, mandò improvvisamente al tappeto Foreman dopo un incontro passato a difendersi alle corde. Cambio di passo e di ritmo, inatteso e quanto mai efficace, quello di Monti; che, proprio come Ali, sembra ora colpire gli avversari «leggero come una farfalla pungente come un'ape» [...].

Occorre riconoscere a Monti una grande abilità comunicativa [...].

Monti ha dimostrato di saperci fare con un'arte che ogni buon leader, al di là della vuota retorica dell'autenticità, dovrebbe almeno saper maneggiare: l'arte di recitare [...].

Facendo eco al berlusconiano «scendere in campo», Monti ha detto di voler «salire in politica».

Perché la politica è cosa nobile: sta in alto; e che richiede sacrifico [sic]: la strada è tutta in salita $[\ldots]$.

Nel momento in cui Monti è salito in politica ha dimostrato di saper cambiare registro comunicativo e linguistico. La comunicazione elitaria ha assunto tonalità popolari. Monti ha abbandonato il registro d'élite, il discorso esclusivamente professorale e tecnico che comunicava competenza, distacco e obiettività - ma anche distanza dal popolo/pubblico - per creare un idioma che mescola sapientemente il discorso del professore con ironia e parlar franco. E questo permette di modificare la percezione della sua persona. Monti resta figura autorevole, dunque distante, ma mostra tratti di umanità, attraverso l'ironia, e capacità di appassionarsi, attraverso il parlar franco: e dunque si avvicina al popolo/pubblico. Parla anche al popolo/pubblico. [...]

Qualcuno potrebbe obiettare che questo modo di fare politica è la continuazione del berlusconismo con altri mezzi. In verità questa è la politica. In cui le cose, per dirla con un filosofo inglese del Novecento, si fanno anche, o soprattutto, con le parole.

L'articolo rinuncia all'ironia e alla riproduzione di moduli del parlato (a parte l'unico scivolamento lessicale saperci fare). Il parlar per metafore, invece, è qui usato con grande sapienza, coerentemente con il registro generale medio-alto. La citazione storica rimanda alla strategia dello storytelling (Salmon, 2008), ormai divenuta dominante nella realizzazione di campagne di marketing in tutti gli ambiti, compreso quello elettorale. Questa strategia rappresenta un formidabile strumento di mistificazione della realtà, attraverso la costruzione, attorno ad un personaggio o un evento reale, di una storia vera o verosimile, associata al personaggio o all'evento con un procedimento di tipo allegorico, del tutto soggettivo. La storia associata evoca valori che automaticamente, subliminalmente, vengono attribuiti al personaggio o all'evento in questione.

L'immagine del politico-pugile, già consolidata, come abbiamo visto, nella retorica tanto politica quanto giornalistica, assume qui la dimensione del racconto di uno degli incontri di boxe più famosi e citati della storia. Grazie all'associazione con l'incontro, Monti diviene Muhammad Ali, che, sfavorito contro un avversario molto più potente, George Foreman, vinse l'incontro grazie all'astuzia. 
Il racconto instaura implicitamente alcune corrispondenze simboliche: le parole degli avversari sono violente ma prive di sostanza, cosi come i pugni di Foreman furono forti ma inefficaci; al contrario le parole di Monti sono misurate, quasi difensive, cosi come la tattica di Ali durante l'incontro fu di limitare i danni attendendo che l'avversario si stancasse. Gli avversari sembreranno avere la meglio per tutta la campagna, ma alla fine Monti/Ali rimonterà e vincerà. Tutto grazie al suo linguaggio.

Le qualità di questo linguaggio, nell'analisi del giornalista, sono: la precisione dei riferimenti («piuttosto un attacco mirato»), la capacità di recitate, l'ironia, il parlar franco. Per il giornalista, quindi, espressioni in codice come «tagliare le ali estreme» e «silenziare le frange estreme» sono attacchi chiari e precisi, nei quali è facile rintracciare il nome e il cognome dei personaggi politici oggetto del riferimento. Apprezzabile, inoltre, sarebbe la capacità di Monti di recitare, cioè di impersonare un ruolo, fingere, evidentemente per compiacere, in superficie, porzioni di elettorato che non si riconoscono nelle sue posizioni. Recitare, con un ragionamento a dir poco contorto, diviene addirittura sinonimo di parlar franco, mentre è chiaro che, nel giudizio del giornalista, Monti non ha cambiato le sue posizioni politiche, ma solamente il suo modo di presentarsi («ha dimostrato di saper cambiare registro comunicativo e linguistico»).

Del resto, argomenta alla fine il giornalista, la politica è il regno della parola, non dei fatti; come in teatro, il politico ha davanti a sé un pubblico, che deve portare dalla sua parte raccontando una storia convincente ed emozionante. E il giornalismo non è diverso, come dimostra la strategia dello storytelling, ma anche l'uso dell'allegoria del teatro e altri artifici sparsi nel testo, a partire dall'evocazione del film uscito nelle sale in quel periodo Django unchained, passando per l'ironico new Monti, finendo con l'argumentum ad verecundiam (Gualdo e Dell'Anna, 2004: 62) "per dirla con un filosofo inglese del Novecento». Quest'ultimo, in particolare, risulta usato scorrettamente, visto che non è fornita l'identificazione precisa del filosofo (probabilmente John Austin).

Un ampio ricorso allo stile brillante si nota anche nel seguente articolo, che commenta alcuni usi linguistici di Pier Luigi Bersani:

(3) Pier Luigi Bersani ha riconquistato il centro del ring mediatico. Grazie ad una metafora decisamente hard («l partiti personali sono il cancro della democrazia») è riuscito a ritrovare spazio nelle prime pagine dei giornali e sui social network, al punto che stamattina ha rincarato la dose, specificando che anche il professor Monti è coinvolto nel suo «j'accuse». Il leader del Pd aveva bisogno di una esternazione forte, che fosse capace di togliere il monopolio dei riflettori a Berlusconi e al tempo stesso «parlasse» al proprio elettorato. Il primo obiettivo l'ha sicuramente raggiunto, il secondo non è facilmente misurabile. Ma è l'assunto di partenza che, paradossalmente, chiama in causa chi ha scagliato la prima pietra.

I partiti personali, nella accezione bersaniana, sono anzitutto quelli che inseriscono il nome del leader nel simbolo elettorale. Ma se bastasse una «debolezza» come questa per prendersi l'accusa di essere un untore di cancri, anche il Pd non potrebbe sottrarsi: nel 2008 il Pd chiese 
voti, esponendo il nome di Veltroni nel suo simbolo e allora non risulta che dentro il partito si fossero levate voci discordi.

Naturalmente non c'è nulla di più personalizzato (e di più democratico) della pre-investitura [sic] popolare di un leader attraverso lo strumento delle Primarie. Da questo punto di vista Bersani è il più «americano» dei leader in campo e, tra l'altro, è anche l'unico che esponga il proprio viso nei manifesti appesi su cartelloni, paline e autobus di tutta Italia. Con una identificazione forte tra partito e leader che, sinora, nessuno degli altri leader ha abbracciato. Neppure Berlusconi. Ovviamente il Cavaliere è l'espressione di declinazione tutta speciale del partito personale: un modello sudamericano di leadership solitaria, che in Italia ha ricevuto diversi riscontri elettorali e che il Pd ha tutto il diritto di avversare. Ma se Bersani allarga il tiro oltre Berlusconi, a quel punto non può non esporsi ad una controprova che lo riguarda. Personalmente. (Martini: 18 gennaio 2013)

Le metafore pescano da diversi ambiti del reale: ricorre il ring di Regazzoni (4 gennaio 2013) dallo sport (insieme a «allarga il tiro»); appare l'economia con «rincarare la dose» (a cui si può avvicinare «il monopolio dei riflettori»), la medicina, attirata dalla citazione dell'espressione di Bersani («untore di cancri»). Notevole l'aggettivo con cui viene definita la metafora citata di Bersani, hard: il significato denotativo della parola è noto solamente a chi ha una discreta conoscenza dell'inglese; comunemente, invece, in Italia esso è associato, connotativamente, ai film pornografici. A rendere ancora più elitario il linguaggio sono le citazioni letterarie («nel suo “j’accuse”», «chi ha scagliato la prima pietra») e i riferimenti geopolitici, in realtà fortemente stereotipici, dell'americanità di Bersani e della sudamericanità di Berlusconi.

Nella conclusione dell'articolo, alcuni segnali nella costruzione del discorso sembrano voler mascherare la debolezza dell'argomentazione: i segnali discorsivi naturalmente e ovviamente e il punto anomalo. Quest'ultimo, in particolare, è caricato di un forte valore illocutivo, difficile da decifrare completamente, sia in «nessuno degli altri leader ha abbracciato. Neppure Berlusconi», sia in «una controprova che lo riguarda. Personalmente» (nel quale è coinvolto anche il gioco di parole intorno al concetto di personale).

Il punto anomalo finale, del resto, si inserisce in un periodo perfettamente adatto alla citata descrizione di Orwell (che, però, era riferita al linguaggio della politica):

(4) Ovviamente il Cavaliere è l'espressione di declinazione tutta speciale del partito personale: un modello sudamericano di leadership solitaria, che in Italia ha ricevuto diversi riscontri elettorali e che il Pd ha tutto il diritto di avversare. Ma se Bersani allarga il tiro oltre Berlusconi, a quel punto non può non esporsi ad una controprova che lo riguarda. Personalmente.

Il lessico dello stralcio è fortemente marcato in diastratia: «l'espressione di declinazione», «riscontri elettorali», «avversare», «allarga il tiro», «controprova». Esso si intreccia con la sintassi contorta del secondo periodo, nel quale nel periodo ipotetico è inserita una doppia negazione. Tutto per dire 
che Bersani, polemico verso gli altri leader di partito per i loro atteggiamenti personalistici, ha degli atteggiamenti più personalistici degli altri leader.

Il seguente articolo manifesta un atteggiamento critico nei confronti degli usi linguistici dei politici. Osserviamo che anche in esso emergono un ragionamento contorto e diverse strategie dell'approssimazione:

(5) La parola è come la pallovale in una partita di rugby al punto che il linguaggio risulta inarticolato, incapace di imbastire un discorso. Tanto impoverito, sbiadito da non poter più esprimere significati. Esso si manifesta soltanto con un alternarsi di pulsioni aggressive e di crolli di tensione. Affermazioni e negazioni.

Si arriva a questa obiettiva conclusione seguendo la nostra campagna elettorale [...].

Non c'è ombra di programma nel fiume di parole che arriva all'imminente elettore. Ma un rosario di battute accompagnate da espressioni scettiche, insultanti, sardoniche, ringhiose, sprezzanti, conniventi, ammiccanti, insomma promettenti [...].

E c'è chi sventola anche i vergognosi debiti dello Stato con le aziende private. Si giura che saranno infine saldati [...].

Il linguaggio politico italiano è sempre o spesso servito a nascondere ciò che è semplice e concreto dietro i giri di parole delle astrazioni generali. Italo Calvino, nelle note sul linguaggio politico, scriveva che il diavolo è l'approssimativo. Per lui, nelle genericità, nell'imprecisione di pensiero e di linguaggio, specie se accompagnati da sicumera e petulanza, si poteva riconoscere il diavolo come nemico della chiarezza, sia interiore, sia nei rapporti con gli altri. Il diavolo non era il complicato, ma l'approssimativo. La semplificazione a tutti i costi è infatti faciloneria. Riuscire a definire i propri dubbi, di fronte a problemi complicati, è molto più concreto che qualsiasi affermazione perentoria basata sul vuoto.

[...] ai demoni tradizionali che affollano il tempio della politica si sono aggiunti i mercanti, i guitti, e i giudici spogliatisi troppo in fretta della toga per scendere nell'arena elettorale. (Valli: 8 febbraio 2013)

Il giornalista sostiene innanzitutto che il linguaggio politico nasconda il semplice dietro giri di parole astratti e generali. Lascia intendere che in questo modo si ceda all'approssimazione, vero male di questo ambito della comunicazione; infine identifica l'approssimazione con la semplificazione a tutti i costi.

C'è nel ragionamento una difficoltà logica: il giornalista identifica l'astrazione prima con l'approssimazione e infine con la semplificazione. Semplificare, quindi, appare all'inizio come valore da difendere, messo a rischio dall'astrazione, ma diviene un disvalore alla fine. Il problema deriva dalla citazione, è il caso di dirlo, approssimativa, della autorità («ltalo Calvino, nelle note sul linguaggio politico, scriveva [...]»): Calvino (1980), in realtà, identifica sì l'approssimazione con il diavolo, ma non difende affatto la semplicità in contrapposizione all'approssimazione, proprio perché conclude 
che pretendere la semplicità su questioni che non sono semplici costringe ad essere approssimativi, generici, quindi menzogneri.

Oltre alla difficoltà logica, frutto di quello stesso difetto comunicativo che il giornalista voleva stigmatizzare, il giornalista usa anche alcune tecniche retoriche e testuali dell'approssimazione, e in particolare:

1. Il parlar figurato, che comprende similitudini («come la pallovale»), metafore («ombra di programma», «fiume di parole», «rosario di battute», gli aggettivi riferiti al linguaggio: «impoverito» e «sbiadito»), iterazioni («espressioni scettiche, insultanti, sardoniche, ringhiose, sprezzanti, conniventi, ammiccanti, insomma promettenti»), epiteti che alludono a persone reali senza nominarle («i mercanti, i guitti, e i giudici spogliatisi troppo in fretta della toga per scendere nell'arena elettorale»);

2. La forma impersonale ( SSi arriva a questa obiettiva conclusione», «Non c'è ombra di programma», «E c'è chi sventola», «si giura che»), la perifrasi avverbiale «sempre o spesso», il punto anomalo («crolli di tensione. Affermazioni e negazioni»).

\section{Scelte lessicali dei politici e dei giornali}

Alcuni termini che hanno avuto corso nella campagna elettorale 2013 sono rari o forestieri, dal significato oscuro o eufemizzante. I media hanno accolto e rilanciato questi termini, senza preoccuparsi di glossarli o di chiedere ai politici di spiegarli.

È certo prevedibile che i politici considerino la lingua come uno strumento con cui mascherare le proprie intenzioni, qualora sospettino che queste creerebbero scontento in parte dell'elettorato. Non altrettanto prevedibile, invece, è che i giornali mostrino di possedere lo stesso vocabolario dei politici, per quanto ambiguo e dissimulatore esso sia.

I seguenti esempi illustrano come i politici e i giornali condividano lo stesso lessico tecnico-specialistico; questa condivisione è messa qui in relazione con la coincidenza degli scopi comunicativi tra stampa e politici, come argomentato finora sul versante dell'organizzazione testuale.

Il termine desistenza (soprattutto in combinazione con patto di), nell'accezione specifica che qui interessa, ha una vita ormai ventennale. De Mauro (1999-2007: s.v.) lo registra così: «(TS) polit. in una tornata elettorale, rinuncia di una formazione politica a presentarsi in un determinato collegio per favorirne un'altra con la quale si è stabilito un accordo: patto di d.». L'accezione tecnico-specialistica del termine si è affermata dopo l'entrata in vigore delle leggi elettorali 4 agosto 1993 n. 276 e n. 277, note complessivamente come Mattarellum. 
Come notano Dell'Anna e Lala (2004: 205), le occorrenze di questo termine, nel corpus giornalistico considerato dai due studiosi, calano notevolmente dal 2000 in poi, fino a toccare un minimo di 17 nel 2003. L'archivio di repubblica.it, da me consultato, contiene appena 22 occorrenze di desistenza in 9 anni, dall'1 gennaio 2004 all'1 gennaio 2013. Nei due mesi successivi, però, dal 2 gennaio al 28 febbraio 2013, desistenza raggiunge le 12 occorrenze.

Il termine ha un significato per niente trasparente. La pratica che indica, infatti, si suppone essere sgradita all'elettorato, sempre più infastidito dagli oscuri giochi di potere dei partiti. I media riportano il termine così com'è, senza glossarlo, senza chiedere spiegazioni, senza rilevarne l'oscurità. Le prime attestazioni nel contesto della campagna elettorale 2013 nell'archivio di repubblica.it risalgono al 14 gennaio e sono addirittura tre in un giorno:

(6) Mentre Berlusconi si divide dalla Lega, infatti, Prodi «unisce» i Popolari e la sinistra. E allarga l'alleanza a Rifondazione, attraverso un patto di desistenza. Da lì in poi, il Cavaliere non accetterà più «faccia a faccia», per quasi 10 anni. (Diamanti: 14 gennaio 2013)

(7) Il «dossier» di Franceschini ha un nome: desistenza. È questa sostanzialmente la proposta lanciata dal capogruppo al movimento di Ingroia. Un'altra parola sfortunata nella storia delle coalizioni di centrosinistra. Segnò la prima vittoria di Prodi nel '96, con gli esiti successivi che si conoscono. Ma la posta in gioco è troppo alta e i segni premonitori vanno esorcizzati. (De Marchis: 14 gennaio 2013)

(8) La precisazione di Ingroia segue le dichiarazioni del sindaco di Palermo, Leoluca Orlando: «Dario Franceschini mi ha contattato questa mattina a nome del Pd e mi ha proposto un accordo di desistenza, cioè mi ha chiesto di non presentare le nostre liste in regioni chiave quali la Sicilia, la Campania e la Lombardia». (repubblica.it: 14 gennaio 2013)

L'unica precisazione sul significato del termine, nel terzo stralcio riportato, viene non da un giornalista, ma dallo stesso personaggio politico che l'ha usato, Leoluca Orlando. Notevole è anche l'atteggiamento del giornalista De Marchis, che non solo non spiega il significato della parola, ma aggancia alla stessa anche delle considerazioni storiche. Il riferimento alle elezioni del 1996 («Segnò la prima vittoria di Prodi nel '96, con gli esiti successivi che si conoscono») è difficilmente collocabile nel contesto dell'articolo; difficoltà aumentata dalla costruzione stessa del riferimento: l'affermazione che fu la desistenza a portare Romano Prodi alla vittoria nelle elezioni del 1996 appare, in effetti, troppo perentoria e affrettata. II giornalista suppone non solo che chi legge conosca il significato tecnico di desistenza, ma anche che ricordi quale ruolo questa pratica ebbe nella campagna elet-

\footnotetext{
5. L'ultima occorrenza del termine (ma in senso figurato) precedente a quelle riportate è di ben tre mesi prima: «Oggi come ieri, è nell'attrazione esercitata da capipopolo dai nomi esoterici: Belzebù, Cavaliere, Celeste, e chissà come designeremo i prossimi. Calamandrei chiamò questo disgusto desistenza, contrapponendola alla tuttora necessaria resistenza. Non più eroica, ma pur sempre resistenza: "resistenza in prosa". Ė tardi per simile resistenza? Non è tardi mai per divenire adulti, e sovrani nella coscienza. Per difendere le mura della legge e le sue sentinelle, come si difendono le mura della città» (Spinelli: 17 ottobre 2012).
} 
torale del 1996 (sempre che ne abbia effettivamente avuto uno). Infine non chiarisce quali «esiti successivi» dovrebbero essere conosciuti dal pubblico; evocato, si noti, con la forma impersonale si conoscono. La sostanza e la forma della citazione rientrano nel carattere del giornalista esperto, che ha una conoscenza dei fatti superiore a quella del lettore; una conoscenza che non va trasmessa o condivisa con il lettore, ma solamente sfoggiata.

Significativa per illustrare l'atteggiamento linguistico dei giornali quando trattano di comunicazione politica è anche la vicenda della parola scouting, prestito non adattato dall'inglese così definito da De Mauro: «L'attività di ricercare volti nuovi spec. per la televisione o il cinema | estens., chi svolge tale attività» (De Mauro, 1999-2007: s.v.).

Il termine è prelevato dal gergo dello spettacolo (e dello sport, se lo si vuole distinguere dallo spettacolo) da Pierluigi Bersani, segretario del Pd, e adattato per definire l'atteggiamento che il Pd avrebbe tenuto nei confronti dei futuri parlamentari 5 Stelle:

(9) ROMA - A Grillo dico che non si vince sulle macerie, sulle macerie sta bene solo chi ha i soldi. Se in Parlamento ci saranno i grillini ci sarà da fare uno scouting per capire se intendono essere eterodiretti o partecipare senza vincoli di mandato. Non è campagna acquisti ma li testeremo sui fatti. (repubblica.it: 19 febbraio 2013)

È lo stesso Bersani a glossare il termine («Non è campagna acquisti»); la glossa, però, non spiega che cosa significa il termine, bensì che cosa non significhi. Il vero significato rimane sospeso. Anche qui il giornalista non chiede spiegazioni, sebbene il termine sia stabilmente usato solamente nel senso proprio indicato dal dizionario, come dimostra la ricerca nell'archivio di repubblica.it nel periodo dall'1 gennaio 2012 al 18 febbraio 2013. Delle 26 occorrenze risultanti, tutte hanno a che fare con la ricerca di talenti dello sport o dello spettacolo, non certo di politici disposti a cambiare partito, e solamente una, la seguente, è riconducibile al contesto della politica (il termine è virgolettato):

(10) E visto che Passera ha già molti dossier aperti - dall'Ilva all'Alcoa, dal decreto Sviluppo al patto sulla produttività - si è affidato per lo «scouting» a due fidatissime teste d'uovo. [...] L'altro dioscuro è Alessandro Fusacchia, che Passera ha nominato suo consigliere al ministero. [...] Ha girato per l'Italia ascoltando centinaia di imprenditori per il progetto «Restart Italia», è presidente della Rete per l'Eccellenza nazionale (Rena) e di sicuro la sua agenda si è riempita in queste settimane di conoscenze utili per allargare il network del suo ministro. (Bei: 13 ottobre 2012)

L'archivio di repubblica.it ci consente di seguire la vita di questa accezione del termine anche in seguito, ad elezioni avvenute. Fino a tutta l'estate 2013, l'unico ad usarlo, due volte, è il politico Beppe Grillo, citando quasi alla lettera la dichiarazione di Bersani. II 27 ottobre il termine riappare, usato

6. Notevole nella dichiarazione anche il termine eterodiretto, altro tecnicismo non spiegato, dalla sociologia, che maschera una valutazione tanto ironica quanto negativa sull'autonomia di giudizio dei parlamentari 5 Stelle. 
dal giornalista autore dell'articolo, nella stessa accezione di Bei (13 ottobre 2012): «Giancarlo Galan, presidente della commissione Cultura, al quale spetterà il talent scouting» (Lopapa: 27 ottobre 2013). Galan dovrà cercare nella società civile persone disposte ad entrare in politica in una nuova formazione epigona di Forza Italia. Il significato corrisponde a quello dato da De Mauro (1999-2007: s.v.), ma è applicato al contesto politico. Nel 2014 risultano due attestazioni dell'accezione tecnica, entrambe in discorsi di politici. Ad un anno di distanza, quindi, l'accezione tecnica del termine è ancora appannaggio dei soli politici, che la usano con intento eufemizzante, ma al contempo allusivo. I giornali, dal canto loro, l'hanno sempre registrata passivamente, senza mettere in evidenza la carica allusiva in essa nascosta.

I giornali sono andati ben oltre con un altro termine, l'aggettivo e sostantivo impresentabile. Questa la definizione del termine data da De Mauro: «Di qcs., che non può essere mostrato in pubblico, perché in disordine: una casa i. | di qcn., che non si può presentare in pubblico, perché mal vestito, disordinato e sim.: vestito cosi sei i» (De Mauro, 1999-2007: s.v.).

L'accezione tecnica usata dai politici e dai giornali, non ancora registrata dal dizionario, significa, grosso modo, «incandidabile perché coinvolto in vicende giudiziarie o in dinamiche politicamente e/o moralmente discutibili». Essa sarebbe di coniazione giornalistica, probabilmente di Marco Travaglio: l'attestazione più vecchia che sono riuscito a rintracciare è Travaglio (2001).

L'uso del termine, nell'accezione tecnica evidenziata, precede, quindi, la campagna elettorale qui osservata, né si interrompe con essa. Durante la campagna elettorale, però, il termine è circolato con insistenza (18 attestazioni nell'archivio di repubblica.it dal 28 dicembre 2012 al 28 febbraio 2013) in seguito alla promulgazione del decreto legislativo 31 dicembre 2012 n. 235 (noto come Legge Severino), ma anche a causa della pressione moralizzatrice esercitata dall'opinione pubblica (sospinta da alcuni media e da alcuni attori politici come il Movimento 5 Stelle).

L'accezione del termine contiene un implicito giudizio di condanna morale nei confronti dei soggetti così definiti, non scisso da una punta di ironia. Veicolare tale implicito è stato ritenuto vantaggioso dai politici, che hanno mutuato il termine per dissociarsi da decisioni del proprio partito o dagli schieramenti avversari. Solo due esempi:

(11) ROMA - Bisogna capire dove sta andando e che cosa vuole essere il Pdl. lo ho detto cosa non voglio: non voglio più un partito di plastica, non voglio impresentabili in lista, non voglio un partito che non discute e non condivide decisioni, non voglio un partito di colonnelli, non voglio i parlamentari scelti da cinque persone in una stanza. (ilmessaggero.it: 10 dicembre 2012)

(12) ROMA - Il liberista Oscar Giannino, con riferimento alla destra berlusconiana che giudica impresentabile e inaffidabile, cita il Mahatma Gandhi a proposito delle sfide impossibili: «Prima ti ignorano, poi ti deridono, poi ti combattono. Poi vinci...». (Martirano: 8 febbraio 2013) 
Allo stesso modo l'implicito è stato sfruttato dai giornalisti, provocando ogni volta uno slittamento non segnalato del discorso dal piano informativo a quello argomentativo: definire impresentabile un politico, infatti, confonde l'incandidabilità stabilita per legge (ai sensi della Legge Severino) con il giudizio, opinabile, sulla condotta morale della persona, per di più costruito in modo ironico. Due esempi di uso brillante del termine: «Condannati e indagati in lista: ecco gli impresentabili di Bersani» (Fontana: 16 gennaio 2013);

(13) Situazione delicata, sono ore decisive, però la partita è aperta. Su un piatto della bilancia c'è il suo curriculum da «impresentabile» (l'ex sottosegretario nato a Casal di Principe 53 anni fa e imparentato con il boss Giuseppe Russo detto Peppe o Padrino, è imputato in due processi per «concorso in associazione mafiosa» e destinatario di due richieste di arresto non autorizzate dal Parlamento); sull'altro piatto ci sono almeno trentamila voti, c'è il controllo quasi militare di tessere e consenso del Pdl in Campania. (Roncone: 15 gennaio 2013)

Nel secondo estratto, il termine, virgolettato, è inserito all'interno della metafora sui piatti della bilancia, conclusa con l'aggettivo ulteriormente metaforico militare, che nasconde un riferimento in parte ironico, in parte fin troppo serio, vista l'elencazione appena fatta.

Il termine, usato in questa accezione, è usato con lo stesso scopo comunicativo dei politici: coinvolgere il pubblico sul piano emotivo, infarcendo il proprio discorso di opinioni non argomentate o mascherate come fatti, espresse in modo vago e ammiccante. Si ripropone, dunque, la questione della funzione che i giornali italiani attribuiscono al linguaggio nel momento in cui commentano l'attualità politica: essi indulgono evidentemente ad un uso linguistico finalizzato ad una funzione non informativa, ma nascostamente valutativa e partigiana.

\section{Conclusioni}

In questo intervento ho cercato di ricavare da commenti giornalistici (da giornali on line) sulla campagna elettorale 2013 alcuni tratti ideologici relativi alla lingua diffusi tra i giornalisti.

Dall'analisi è emerso che i giornalisti, mentre esprimono giudizi di vario genere sul linguaggio dei politici, mostrano, attraverso le strategie testuali adottate, di prefiggersi gli stessi scopi comunicativi dei politici: abbassare la complessità concettuale della comunicazione in favore dell'emotività diretta e della partecipazione irriflessa del lettore.

In altre parole, nell'ideologia linguistica dei giornali, il linguaggio non ha la funzione di rendere più chiare e precise le dichiarazioni dei politici, per loro natura approssimative e opinabili, ma serve ad aggirare la razionalità dei lettori, proprio come fa il linguaggio dei politici, coinvolgendoli sul piano emotivo con diversi strumenti retorici e testuali. Il ricorso allo stile brillante è, dunque, una scelta di ideologia linguistica, in quanto lo stile brillante mette in secondo piano la notizia in sé e sposta l'attenzione sul modo in cui la notizia è costruita. 
Questo avviene o perché il giornale è allineato con un partito, di cui rappresenta un organo di propaganda, o perché il giornalista ama mostrarsi come esperto del sottocodice linguistico del politichese, puntando a stupire i lettori con la sua conoscenza, o a incuriosirli con costruzioni affabulatorie, oppure a imporre delle tesi attraverso meccanismi retorici, o persino a confermare le opinioni che i lettori già possiedono.

La ricerca ha avuto come oggetto anche articoli non strettamente di commento al linguaggio dei politici. In questi articoli sono stati rintracciati esempi di lessico tecnico-specialistico che i politici e i giornalisti si scambiano osmoticamente; un lessico eufemizzante, poco perspicuo, allusivo, che maschera i fatti e le opinioni e veicola implicature allusive. Anche su questo fronte si conferma che la funzione del linguaggio usato nella cronaca politica, all'interno dell'ideologia radicata nella stampa italiana sulla propria lingua, coincide con la funzione che i politici stessi attribuiscono alla propria lingua.

Uno sviluppo possibile per questa ricerca è l'indagine sui giudizi espressi da gente comune sugli usi linguistici (parole, espressioni, abiti comunicativi) dei politici. Questo tipo di indagine metterebbe in luce alcune caratteristiche delle ideologie linguistiche diffuse tra la gente comune, un oggetto finora poco considerato in Italia, ma che produce un impatto sociale di primaria importanza. 


\section{Bibliografia}

\section{Fonti primarie}

Bei, Francesco (2012), «l piani di Passera: lista civica collegata all'Udc, poi patto di governo con il Partito democratico», la Repubblica.it, 13 dicembre, disponibile su www.repubblica.it/politica/2012/10/13/news/road_map_passera-44434354/?ref=search. [Sito consultato l'8 marzo 2014.]

De Marchis, Goffredo (2013), "Bersani contro Casini: "Vuole comandare senza voti"», la Repubblica.it, 14 gennaio, disponibile su www.repubblica.it/politica/2013/01/14/news/bersani_casini-50487556/?ref=search. [Sito consultato l'8 ottobre 2014.]

Diamanti, Ilvo (2013), «La video-politica di Silvio Munchausen», la Repubblica.it, 14 gennaio, disponibile su www.repubblica.it/politica/2013/01/14/news/mappe_video_politica-50487555/?ref=search. [Sito consultato l'8 ottobre 2014.]

Fontana, Emanuela (2013), «Condannati e indagati in lista: ecco gli impresentabili di Bersani», il Giornale.it, 16 gennaio, disponibile su www.ilgiornale.it/news/interni/condannati-e-indagati-lista-ecco-impresentabili-bersani-875368.html. [Sito consultato l'8 ottobre 2014.]

Giulietti, Beppe (2010), «Chi presenta gli impresentabili?», il Fatto Quotidiano.it, 13 ottobre, disponibile su www.ilfattoquotidiano.it/2010/10/13/chi-presenta-gli-impresentabili/71375/. [Sito consultato l'8 marzo 2014.]

Ilmessaggero.it (redazione) (2012), «Meloni pronta a lasciare il Pdl: "Non trovo stimoli per rimanere”», /l Messaggero.it, 10 dicembre, disponibile su www.ilmessaggero.it/PRIMOPIANO/POLITICA/ meloni_pronta_a_lasciare_il_pdl_laquo_non_trovo_stimoli_per_rimanere_raquo/notizie/237115.shtml. [Sito consultato l'8 ottobre 2014.]

Ilsole24ore.com (redazione) (2013), «Berlusconi pifferaio, Monti mascalzone, Bersani Gargamella. A un mese dalle elezioni è una campagna piena di insulti», // Sole 24 Ore, 20 gennaio, disponibile su www.ilsole24ore.com/art/notizie/2013-01-20/berlusconi-pifferaio-monti-mascalzone-173135.shtml?uuid=Ab8pfGMH. [Sito consultato l'8 marzo 2014.]

Lopapa, Carmelo (2013), «L'ultima svolta di Berlusconi: “Possiamo candidare Marina”», la Repubblica.it, 27 ottobre, disponibile su www.repubblica.it/politica/2013/10/27/news/l_ultima_svolta_di_berlusconi_possiamo_candidare_marina-69542500/?ref=search. [Sito consultato l'8 ottobre 2014.]

Lucarelli, Ottavio (2013), «Casini lancia una bordata al Cavaliere “Un buffone, roba da circo Togni”», la Reppublica.it, 20 febbraio, disponibile su http://ricerca.repubblica.it/repubblica/archivio/repubblica/2013/02/20/casini-lancia-una-bordata-al-cavaliere-un.html. [Sito consultato l'8 marzo 2014.] 
Martini, Fabio (2013), «"Le liste personali sono un cancro”. Bersani e la tattica delle parole forti», La Stampa.it, 18 gennaio, disponibile su www.lastampa.it/2013/01/18/italia/politica/le-liste-personali-sono-un-cancro-bersani-e-la-tattica-delle-parole-forti-1SYZvryrEAIYpkgRRStbzJ/pagina.html. [Sito consultato l'8 marzo 2014.]

Martirano, Dino (2013), «Gandhiano, liberista e dandy: il caso Giannino», Corriere della Sera.it, 8 febbraio, disponibile su www.corriere.it/politica/speciali/2013/elezioni/notizie/giannino-ritratto_0e031a88-71ba-11e2-8d40-790077d2d105.shtml. [Sito consultato l'8 ottobre 2014.]

Mauro, Angela (2013), «Elezioni 2013. Bersani apre la "fase 2" della campagna elettorale: proposte per economia e lavoro. Nel weekend tappa in Piemonte, dove sarà anche Renzi. Ma i due si vedranno solo allo stadio...», L'Huffington Post, 7 febbraio, disponibile su www.huffingtonpost. it/2013/02/07/elezioni-2013-bersani-apre-fase-due-campagna-elettorale-proposte-economiche-lavoro-domani-altro-round-a-torino_n_2637807.html. [Sito consultato l'8 marzo 2014.]

Pica, Paolo (2013), «Bersani: "Mps, se ci attaccano li sbraniamo. Ma non crediamo di aver già vinto"», Corriere della Sera.it, 26 gennaio, disponibile su www.corriere.it/politica/13_gennaio_26/ dalema-mps-monti-monaci_88fd384e-67a2-11e2-8a76-cd5e3242e53f.shtml. [Sito consultato l'8 marzo 2014.]

Rainews24.rai.it (redazione) (2013), «Bersani: non è Tangentopoli ‘92 ma la destra ci consegna catastrofe morale», 15 febbraio, disponibile su www.rainews24.rai.it/IT/news.php?newsid=174945. [Sito consultato l'8 marzo 2014.]

Regazzoni, Simone (2013), «ll nuovo linguaggio del professore scatenato», I/ Secolo XIX, 4 gennaio, disponibile su www.ilsecoloxix.it/p/italia/2013/01/04/APq2yLNE-professore_scatenato_linguaggio.shtml. [Sito consultato l'8 marzo 2014.]

Repubblica.it (redazione) (2013a), «Ingroia: "Nessun patto di desistenza col Pd" "Monti vuole togliere le tasse che ha messo"», la Repubblica.it, 14 gennaio, disponibile su www.repubblica.it/politica/2013/01/14/news/bersani_al_washington_post_con_monti_niente_favori_ma_patto_ per_le_riforme-50515866/?ref=search. [Sito consultato l'8 marzo 2014.]

Repubblica.it (redazione) (2013b), «Monti: "Berlusconi pifferaio magico, sulle tasse aveva illuso anche me” », 14 gennaio, disponibile su www.repubblica.it/politica/2013/01/14/news/monti_situazione_grave_colpa_di_chi_ha_governato_prima-50540745/. [Sito consultato l'8 marzo 2014.]

Repubblica.it (redazione) (2013c), «Bersani: “Con grillini confronto in Parlamento. Spartizione Pd-Pdl? Ipotesi lunare"», la Repubblica.it, 19 febbraio, disponibile su www.repubblica.it/speciali/politica/elezioni2013/2013/02/19/news/bersani_con_i_grillini_faremo_scouting-52965563/?ref=search. [Sito consultato l'8 marzo 2014.]

Rho, Roberto (2012), «Formigoni cancelli il listino della vergogna», la Repubblica.it, 15 gennaio, disponibile su www.ricerca.repubblica.it/repubblica/archivio/repubblica/2012/01/15/formigoni-cancelli-il-listino-della-vergogna.html?ref=search. [Sito consultato l'8 marzo 2014.] 
Roncone, Fabrizio (2013), «Cosentino e gli "impresentabili". Papa e Laboccetta: va candidato. Con noi», Corriere della Sera.it, 15 gennaio, disponibile su www.corriere.it/politica/13_gennaio_15/ cosentino-e-gli-impresentabili-papa-e-laboccetta-va-candidato-con-noi-fabrizio-roncone_1488feac-5ede-11e2-8d79-cb6cdb3edff8.shtml. [Sito consultato l'8 marzo 2014.]

Severgnini, Beppe (2013), "Con le battute non si governa», Corriere della Sera.it, 21 febbraio, disponibile su www.archiviostorico.corriere.it/2013/febbraio/21/CON_BATTUTE_NON_GOVERNA_co_0_20130221_26c3e03c-7bee-11e2-91ba-4fccd0ebf5fe.shtml. [Sito consultato l'8 marzo 2014.]

Simili, Bruno (2013), «I grillini a Roma da attivisti a politici», la Repubblica.it, 3 febbraio, disponibile su www.ricerca.repubblica.it/repubblica/archivio/repubblica/2013/02/03/grillini-roma-da-attivisti-politici.html?ref=search. [Sito consultato l'8 marzo 2014.]

Spinelli, Barbara (2012), «Una legge vera contro i corrotti», la Repubblica.it, 17 ottobre, disponibile su www.repubblica.it/politica/2012/10/17/news/legge_corrotti-44685169/?ref=search. [Sito consultato l'8 marzo 2014.]

Tg24.sky.it (redazione) (2013), «Berlusconi: "Monti, Casini e Fini? Un trio sciagura”», 5 gennaio, disponibile su www.tg24.sky.it/tg24/politica/2013/01/05/berlusconi_videochat_rapporti_lega_ mario_monti_trio_sciagura.html. [Sito consultato l'8 marzo 2014.]

Tg1.rai.it (redazione) (2013), «Berlusconi: “io un po' strega, sento che vinceremo"», 1 febbraio, disponibile su www.tg1.rai.it/dl/tg1/2010/articoli/Contentltem-5faa9eec-b68b-4443-a4f858b94910791a.html?refresh_ce. [Sito consultato l'8 marzo 2014.]

Travaglio, Marco (2001), «Sconsigli per le liste: le pagine gialle degli impresentabili», MicroMega, $n^{\circ} 2$ (L'Italia che rischiamo), p. 55-70.

Valli, Bernardo (2013), «Se le elezioni diventano un'asta», la Repubblica.it, 8 febbraio, disponibile su http://ricerca.repubblica.it/repubblica/archivio/repubblica/2013/02/08/se-le-elezioni-diventano-unasta.html. [Sito consultato l'8 marzo 2014.]

\section{Fonti secondarie}

Antenore, Marzia, Loris Di Giammaria e Serena Gennaro (2013), «TweeTelection. Tra salotti televisivi e arene digitali», in Mario Morcellini, Marzia Antenore e Christian Ruggiero (ed.), Talk\&Tweet. La campagna elettorale 2013 tra Tv e Twitter, Maggioli, Santarcangelo di Romagna, p. 189-208.

Calvino, Italo (1980), Una pietra sopra. Note sul linguaggio politico, Torino, Einaudi.

Censis (2013), L'evoluzione digitale della specie. Undicesimo Rapporto Censis/Ucsi sulla comunicazione, Milano, Franco Angeli.

Dardano, Maurizio (1994), «La lingua dei media», in Valerio Castronovo e Nicola Tranfaglia (ed.), La stampa italiana nell'età della TV. 1975-1994, Laterza, Bari, p. 207-235.

Dell'Anna, Maria Vittoria e Pierpaolo Lala (2004), Mi consenta un girotondo. Lingua e lessico nella Se- 
conda Repubblica, Galatina, Congedo.

De Mauro, Tullio (ed.) (1999-2007), GRADIT. Grande dizionario italiano dell'uso, Torino, UTET.

Gualdo, Riccardo e Maria Vittoria Dell'Anna (2004), La faconda Repubblica. La lingua della politica in Italia (1992-2004), Lecce, Manni.

Human Highway/Liquida (2011), Il nuovo sistema dell'informazione online. Editori tradizionali, Blog e social content, disponibile su www.slideshare.net/liquida/levoluzione-dellinformazione-online. [Sito consultato l'8 marzo 2014.]

Loporcaro, Michele (2005), Cattive notizie. La retorica senza lumi dei mass media italiani, Milano, Feltrinelli.

Orwell, George (1946), «Politics and the English Language», Horizon, vol. 13, n 76, p. 252-265.

Salmon, Christian (2008), Storytelling. La fabbrica delle storie, Milano, Fazi. [Titolo originale: Storytelling. La machine à fabriquer des histoires et à formater les esprits, Paris, Éditions La Découverte, 2007. Tradotto dal francese da Giuliano Gasparri.]

Woolard, Kathryn A. (1998), «Introduction. Language ideology as a field of inquiry», in Bambi Schieffelin, Kathryn A. Woolard e Paul V. Kroskrity (ed.), Language ideologies. Practice and theory, New York/Oxford, Oxford University Press, p. 3-47. 